\title{
A secure framework exploiting content guided and automated algorithms for real time video searching
}

\author{
Dimitris Halkos • Nikolaos Doulamis • \\ Anastasios Doulamis • Angelos Yannopoulos • \\ Theodora Varvarigou • George Mourkousis • \\ Katerina Tsiara
}

Published online: 27 January 2009

(C) Springer Science + Business Media, LLC 2009

\section{Erratum to: Multimed Tools Appl DOI 10.1007/s11042-008-0234-z}

The original version of this article unfortunately contained a mistake. Angelos Yannopoulos, Theodora Varvarigou, George Mourkousis and Katerina Tsiara were not listed among the authors.

The online version of the original article can be found at http://dx.doi.org/10.1007/s11042-008-0234-z

D. Halkos $(\bowtie) \cdot$ N. Doulamis $\cdot$ A. Yannopoulos $\cdot$ T. Varvarigou $\cdot$ G. Mourkousis $\cdot$ K. Tsiara Department of Electrical \& Computer Engineering, National Technical University of Athens, Athens 157 73, Greece

e-mail: dhalk@telecom.ntua.gr

N. Doulamis

e-mail: ndoulam@cs.ntua.gr
A. Yannopoulos
e-mail: ang@telecom.ntua.gr
T. Varvarigou
e-mail: dora@telecom.ntua.gr
G. Mourkousis
e-mail: georgemr@telecom.ntua.gr
K. Tsiara
e-mail: tsiara@telecom.ntua.gr
A. Doulamis
Laboratory of Decision Support Systems, Technical University of Crete,
Polytechnioupolis, Chania, Greece
e-mail: adoulam@cs.ntua.gr 